\title{
Manifestaciones orales asociadas a la infección por el VIH/sida en la etapa de expansión de la terapéutica antirretroviral: una década en revisión (2000-2010)
}

\author{
Oral Manifestations Associated to HIV Infection under ART/ \\ HAART: Review of a Decade (2000-2010)
}

John Harold Estrada Montoya Odontólogo, Universidad Nacional de Colombia, Bogotá, Colombia. Magíster en Educación, Pontificia Universidad Javeriana, Bogotá, Colombia. Doctor en Salud Pública, Profesor titular

Director Departamento de Salud Colectiva, Facultad de Odontología, Universidad Nacional de Colombia.

Diego Alejandro Ramírez Rojas Odontólogo, estudiante de

Especialización en Ortodoncia, Universidad Nacional de Colombia, Bogotá, Colombia.

Artículo de reflexión, subproducto de la línea de investigación sobre

"Lesiones orales asociadas a la infección por el VIH”, apoyada por el grupo de investigación

Salud Colectiva (clasificación A de Colciencias) del Departamento

de Salud Colectiva, Facultad de Odontología, Universidad Nacional de Colombia. Este proyecto no contó con financiación externa y corresponde a un trabajo final para optar el título de pregrado en Odontología realizado entre julio del 2010 y julio del 2011

CÓMO CITAR ESTE ARTÍCULO Estrada JH, Ramírez DA. Manifestaciones orales asociadas a la infección por el VIH/sida en la tapa de expansión de la terapética antirr)

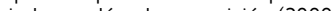
2010). Univ Odontol. 2014 Jul-Dic; 33(71): _. http://dx.doi. org/10.11144/Javeriana.uo33-71.moai

doi:10.11144/Javeriana.uo33-71.moai

Recibido para publicación: 17/03/2014 Aceptado para publicación: 21/09/2014

Disponible en: http://www.javeriana.edu.co/ universitasodontologica

\section{RESUMEN}

Objetivo: identificar los indicadores epidemiológicos de manifestaciones orales asociadas con la infección por virus de inmunodeficiencia humana $(\mathrm{VIH})$ en la etapa de expansión terapéutica antirretroviral. Métodos: se revisó literatura en las bases bibliográficas disponibles en la Universidad Nacional de Colombia. Se utilizó un proceso de revisión con base en la evidencia. Cumplieron los criterios de inclusión 52 artículos publicados entre 2000 y 2010. Resultados: después de la introducción de tratamiento antirretroviral de gran actividad, los estudios proveen evidencia consistente en cuanto a: 1) menor prevalencia, gravedad o extensión de candidosis oral, leucoplasia vellosa y enfermedad periodontal asociada al $\mathrm{VIH}$; 2) decremento global, aunque todavía prevalencia alta de sarcoma de Kaposi en África y Latinoamérica, quizás por desigual distribución geográfica del herpesvirus humano 8, y 3 ) incremento en verrugas orales y úlceras asociadas a los antirretrovirales. Conclusiones: este artículo de reflexión respalda el beneficio de la ampliación de los regímenes de antirretrovirales en todo el mundo y una importante reducción de las manifestaciones orales asociadas al VIH aunque un menor descenso en los países pobres. Los retos para la odontología están en identificar y tratar adecuadamente a las personas $\mathrm{VIH}+$ especialmente en países con recursos limitados.

\section{PALABRAS CLAVE}

antirretrovirales; Colombia; epidemiología; infecciones por VIH; manifestaciones bucales; revisión de la literatura

\section{ÁREAS TEMÁTICAS \\ Epidemiología oral; medicina oral}

\section{ABSTRACT}

Objective: To identify epidemiological indicators of oral manifestations associated to HIV/AIDS. Methods: This is an evidence-based review of literature carried out using the bibliographic databases available at the National University of Colombia. Fifty two articles published between 2000 and 2010 met the inclusion criteria. Results: After the introduction of the high activity antiretroviral therapy (HAART), studies provide consistent evidence of: (1) lesser prevalence, severity, or extent of oral candidiasis, oral hairy leukoplakia and HIV-related periodontal disease; (2) global decrease but nevertheless high prevalence of Kaposiss sarcoma in Africa and Latin America due to the unequal geographical distribution of human herpesvirus 8; and (3) increases in oral warts and antiretroviral-related (ARV) ulcers. Conclusions: The reports reviewed provide evidence of the benefits of scaling-up ART/ HAART regimens worldwide and an important reduction of oral manifestations associated to HIV-infection (except oral warts and ARV-related ulcers), although in a lesser degree in the underdeveloped countries. Challenges lie in the role of oral health care workers in identifying patients who are HIV-positive and in effectively managing their oral and systemic conditions, particularly in resource-poor settings.

\section{KEYWORDS}

antiretroviral therapy; Colombia; epidemiology; HIV infection; literature review; oral manifestations

THEMATIC FIELDS

Oral epidemiology; oral medicine 


\section{INTRODUCCIÓN}

Son numerosas las consecuencias en los órdenes económico, clínico, terapéutico y, especialmente, social, derivadas de la infección por el virus de inmunodeficiencia humana (VIH) y el sida. Esto ha llevado a desarrollar propuestas novedosas de investigación en salud e incluso a transformaciones en la epidemiología clásica. Lo anterior obedece a que, concomitantemente con la epidemia, reemergieron infecciones oportunistas y neoplasias y aparecieron enfermedades no informadas antes en seres humanos, que tienen fuertes repercusiones sociales y humanas (1-5). El reconocimiento de las lesiones orales asociadas al VIH tiene gran importancia, ya que pueden representar el primer signo de la infección, servir como predictores de inmunosupresión y del desarrollo de sida en pacientes infectados, y alertar sobre el síndrome de reconstitución inmune al inicio de la terapia o de la falla terapéutica de los medicamentos antirretrovirales (ARV) en personas que los reciben. La detección precoz de lesiones orales asociadas permite ofrecer un tratamiento oportuno a los pacientes, lo cual redunda en una mejoría de su calidad de vida y, por ende, de toda la sociedad (6-14).

En 1994, los documentos oficiales de la Organización Panamericana de la Salud (OPS), especialmente los relacionados con las políticas de salud oral para todo el continente americano, recomendaban poner en marcha programas para disminuir el costo social de la pandemia por VIH. Dichos programas debían implementar planes de prevención fundamentados en educación, vigilancia epidemiológica y provisión de preservativos o condones. Además, sugería a las facultades de odontología incluir en los currículos el manejo de la infección por $\mathrm{VIH}$. A los investigadores les solicitó esforzarse por determinar los perfiles de morbilidad oral asociados al VIH, su prevalencia, consecuencias y repercusiones, así como los protocolos para mejorar la atención a las personas que viven con el virus. Así se podrían aunar esfuerzos en la lucha contra la pandemia y su propagación y disminuir el estigma a las personas afectadas (5,15-17).

En relación con las entidades orales asociadas a la infección por el VIH, hasta este momento se han publicado muchos informes. Se destaca el estudio de Pindborg (18), quien presenta la primera clasificación de las manifestaciones orales, agrupadas para esa época según el agente etiológico en infecciones fúngicas, bacterianas, virales, trastornos neurológicos, lesiones neoplásicas y un gran grupo de lesiones de etiología desconocida, donde se colocaban todas aquellas entidades hasta ahora desconocidas $(5,17)$. Más tarde, en 1991, Scully y colaboradores $(19,20)$ revisaron la clasificación existente y propusieron dos grandes grupos de acuerdo con la frecuencia de presentación, a fin de ayudar a los odontólogos en el diagnóstico. Además, hicieron un llamado a concentrar la atención y difusión de información en las lesiones orales más frecuentes, esto es: candidosis seudomembranosa, gingivitis y periodontitis asociadas al $\mathrm{VIH}$, leucoplasia vellosa, sarcoma de Kaposi y linfoma no Hodgkin (5).

También en 1991, debido a las discrepancias que se empezaban a identificar regionalmente, se ajustó la clasificación. Se tomó el grado de asociación entre la infección por el VIH y la presencia de lesiones orales como referente. La nueva clasificación se planteaba así: lesiones fuertemente asociadas, moderadamente asociadas y observadas ocasionalmente en infección por el VIH (21). Sin embargo, al año siguiente (1992), el centro colaborativo de la Organización Mundial de la Salud (OMS) para lesiones orales asociadas al VIH, con base en esta clasificación, publicó una nueva versión resultante del consenso entre los grupos de investigación de los países del primer mundo. La clasificación de la OMS rige actualmente para la realización y publicación de estudios epidemiológicos en todo el mundo, y a pesar del paso de los años, es ampliamente utilizada, difundida y, por su gran versatilidad y utilidad, parece destinada a perdurar aún durante largo tiempo (tabla 1) (5,22).

TABLA 1

ClasificaCión aCtuAL de LAS LESIONES ORALES ASOCIADAS A LA INFECCIÓN VIH

\begin{tabular}{llll}
\hline & $\begin{array}{c}\text { Lesiones frecuentemente } \\
\text { asociadas }\end{array}$ & $\begin{array}{c}\text { Lesiones asociadas menos } \\
\text { frecuentemente }\end{array}$ & $\begin{array}{c}\text { Lesiones observadas } \\
\text { ocasionalmente }\end{array}$ \\
\hline Tipo & - Candidosis oral (eritematosa, & . Ulceración atípica & $\cdot$ Infecciones bacterianas por: \\
de & seudomembranosa) & . Afección de glándulas salivares & Actinomyces israelii, \\
lesión & $\cdot$ Leucoplasia vellosa & (xerostomía, agrandamiento & Enterobacter cloacae, \\
& & uni o bilateral de glándulas & Escherichia coli, \\
& & mayores $)$ & Klebsiella pneumoniae
\end{tabular}




\begin{tabular}{|c|c|c|c|}
\hline $\begin{array}{l}\text { Tipo } \\
\text { de } \\
\text { lesión }\end{array}$ & $\begin{array}{l}\text { Enfermedad periodontal } \\
\text { (eritema gingival linear, } \\
\text { gingivitis ulcerativa } \\
\text { necrotizante, periodontitis } \\
\text { ulcerativa necrotizante) } \\
\text { - Sarcoma de Kaposi } \\
\text { - Linfoma no Hodgkin }\end{array}$ & $\begin{array}{l}\text { Púrpura trombocitopénica } \\
\text { Infección por: herpes virus, } \\
\text { papiloma virus (hiperplasia } \\
\text { epitelial focal, verruga vulgar, } \\
\text { condiloma acuminado), varicela } \\
\text { zoster virus (varicela y herpes } \\
\text { zoster) } \\
\text { · Infecciones bacterianas } \\
\text { por: Mycobacterium avium } \\
\text { intracellulare } \\
\text { M. tuberculosis } \\
\text { Hiperpigmentación melánica } \\
\text { Estomatitis ulcerativa } \\
\text { necrotizante }\end{array}$ & $\begin{array}{l}\text { - Enfermedad del arañazo de gato } \\
\text { · Infecciones fúngicas por: } \\
\text { Cryptococcus neoformans } \\
\text { Geotrichum candidum } \\
\text { Histoplasma capsulatum } \\
\text { Mucorales (mucormicosis/ } \\
\text { zygomicosis) } \\
\text { Aspergillus flavus } \\
\text { - Reacciones a drogas } \\
\text { (úlceras, eritema multiforme, } \\
\text { epidermólisis tóxica) } \\
\text { - Trastornos neurológicos } \\
\text { (parálisis facial, neuralgia del } \\
\text { trigémino) } \\
\text { - Estomatitis aftosa recurrente } \\
\text { - Infecciones por citomegalovirus } \\
\text { - Molusco contagioso }\end{array}$ \\
\hline
\end{tabular}

Fuente: EC-Clearinghouse on oral problems related to HIV infection and WHO collaborating centre on oral manifestations of the immunodeficiency virus. Classification and diagnostic criteria for oral lesions in HIV infection. J Oral Pathol Med 1993; 22: 289-91, citado por: Estrada JH. Lesiones orales asociadas con la infección por VIH/Sida en individuos sin tratamiento antirretroviral en el Hospital San Juan de Dios de Bogotá. Biomédica. 2001; 21: 333-44.

\section{Criterios diagnósticos}

Los criterios para el diagnóstico de las lesiones fuertemente asociadas a la infección por el VIH corresponden a las pautas del sistema estandarizado de la OMS/OPS para registrar lesiones orales posiblemente relacionadas con infección VIH (Ginebra, 1989); los criterios derivados de talleres organizados por la OMS/OPS y otras asociaciones científicas en varias oportunidades, y el consenso mencionado, ampliamente utilizado desde entonces (5,7-9,22-24). Dichos criterios son:

Candidosis. Se manifiesta de tres modos. El primero es la candidosis seudomembranosa, que presenta manchas (placas) blanco-amarillentas de aspecto cremoso, localizadas en cualquier parte de la cavidad bucal que pueden ser raspadas con cierta dificultad y dejan una superficie eritematosa y dolorosa con tendencia al sangrado. Los pacientes pueden experimentar irritación y ardor en la boca. La segunda es la candidosis eritematosa, que muestra áreas rojas y atróficas usualmente localizadas en el paladar o dorso de la lengua (donde se aprecia como una zona despapilada y lisa) y, ocasionalmente, en la mucosa bucal con sintomatología o sin esta en el paciente. La tercera es la queilitis angular, que se presenta como grietas, fisuras y ulceraciones uni o bilaterales de la comisura labial, con un patrón radiado y que es generalmente asintomática (25-28).
Leucoplasia vellosa. Se presenta en forma de áreas blanco-grisáceas, ligeramente elevadas, uni o bilaterales en los márgenes de la lengua. No se puede remover y se observa un patrón corrugado vertical que sigue el patrón del epitelio lingual. Las lesiones pueden extenderse en las superficies ventral o dorsal de la lengua donde toman un aspecto liso. También pueden extenderse hasta la mucosa bucal. El rango de las lesiones va desde sutiles áreas blancas translúcidas hasta densas placas blancas asintomáticas $(25,29-31)$.

Enfermedad periodontal. Se revela de tres maneras. Primero, el eritema gingival linear, que es una banda roja (eritematosa) de 2-3 $\mathrm{mm}$ de ancho a lo largo del margen gingival, con sangrado gingival ocasional, desproporcionadamente enrojecida en relación con el nivel de placa blanda o calcificada observado; no muestra ulceraciones, bolsas, ni pérdida de adherencia epitelial; puede extenderse a la encía adherida. Segundo, la gingivitis ulcerativa necrotizante, que consiste en la destrucción de una o más papilas interdentales con apariencia típica de "sacabocados", necrosis, hemorragia y halitosis características y sensación de presión y dolor interdental como en "cuña de madera". Tercero, la periodontitis ulcerativa necrotizante, que se caracteriza por pérdida de tejidos blando y duro como resultado de ulceración o necrosis; presenta exposición, destrucción o secues-

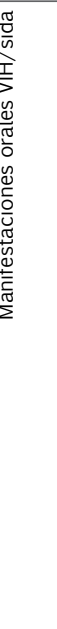


tro de hueso con afectación o pérdida dental, dolor prominente, sangrado espontáneo y progreso rápido $(7,22,25,32-36)$.

Sarcoma de Kaposi. Presenta máculas o pápulas eritematosas violáceas o de color pardo. También se muestra como nódulos con ulceración o sin esta, ubicados preferentemente en el paladar y la encía, aunque pueden aparecer en cualquier lugar de la mucosa (37).

Linfoma no-Hodgkin. Consiste en protuberancias firmes, elásticas, rojas o púrpuras, con o sin ulceración, que afectan encía, paladar y mucosa yugal (38).

El objetivo del presente trabajo fue identificar los indicadores epidemiológicos de manifestaciones orales asociadas con la infección por el VIH en etapa de expansión terapéutica antirretroviral que fueran reportados en la literatura biomédica entre los años 2000 y 2010.

\section{MATERIALES Y MÉTODOS}

Esta investigación es una revisión exhaustiva de la literatura que se efectuó entre julio del 2010 y julio del 2011. Para ello se realizó una búsqueda de artículos en diferentes bases de datos disponibles en la Universidad Nacional de Colombia, sede Bogotá (Science Direct, EBSCO, PubMed, JSTOR, OVID y Hinari). Los términos de la búsqueda fueron Oral lesions AND HIV, Oral manifestations AND HIV, Oral diseases AND HIV y Dentistry AND HIV. Los filtros utilizados incluyeron idioma (inglés, español y portugués), artículos en texto completo y periodo de publicación. Los criterios de inclusión fueron:

- Artículos publicados entre enero del 2000 y diciembre del 2010.

- Artículos que reportaran mínimo dos manifestaciones orales asociadas al $\mathrm{VIH}$.
- Artículos que informaran de la prevalencia.

- Artículos que fueran estudios primarios.

Los criterios de exclusión fueron:

- Artículos publicados antes de enero del 2000 y después de diciembre del 2010.

- Artículos que reportaran solo una manifestación oral asociada al $\mathrm{VIH}$.

- Artículos que no informaran de la prevalencia.

- Artículos que no fueran estudios con pacientes (tipo revisión de la literatura o consenso de expertos).

Un siguiente momento tuvo lugar para refinar aún más la búsqueda. Se revisó la bibliografía de los artículos preseleccionados para acceder a estudios que no hubieran aparecido con las estrategias anteriores. Para acceder a los textos completos en español, portugués e inglés, se empleó Google Académico con los mismos criterios expuestos. Como estrategia complementaria, se recurrió a la búsqueda directa en los sitios en internet de revistas prestigiosas en el tema. Se incluyeron Oral Diseases; Oral Surgery, Oral Medicine, Oral Pathology, Oral Radiology, and Endodontology; Journal of Oral Pathology and Medicine y AIDS.

Los artículos fueron revisados de manera independiente por cada uno de los investigadores y luego de manera conjunta para verificar la clasificación del tipo de estudio y dirimir las dudas hubieran surgido (figura 1).

\section{RESULTADOS Y DISCUSIÓN}

\section{Hallazgos}

Del total de 71 artículos resultantes de la búsqueda inicial se excluyeron 19 que se utilizaron posteriormente para la construcción del marco teórico. Los 52 artículos restantes se clasificaron como se presenta en las tablas 2 a 5.

FIGURA 1

IDENTIIICACIÓN DE LOS ESTUDIOS Y PROCESO DE SELECCIÓN

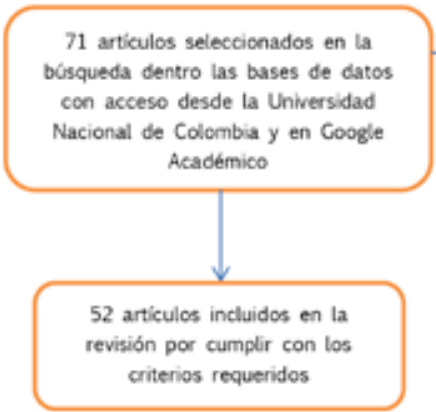

19 articulos excluidos por no cumplir con los criterios relacionados con el objetivo del estudio, pero que presentan utilidad para el cuerpo del marco té́rico 
TABLA 2

NÚMERO DE ARTÍCULOS POR TíTULO DE REVISTA

\begin{tabular}{lc}
\hline \multicolumn{1}{c}{ Revista } & $\begin{array}{c}\text { Número } \\
\text { de }\end{array}$ \\
& \begin{tabular}{l} 
artículos \\
\hline Oral Diseases
\end{tabular} \\
\hline Journal of Oral Pathology and & 9 \\
\hline Medicine & \\
\hline Oral Surgery, Oral Medicine, Oral & 5 \\
Pathology, Oral Radiology, and & \\
\hline Endodontology & \\
\hline $\begin{array}{l}\text { Medicina Oral, Patología Oral y Cirugía } \\
\text { Bucal }\end{array}$ & 3 \\
\hline AIDS Research and Therapy & 2 \\
\hline Avances en Odontoestomatología & 2 \\
\hline JAIDS: Journal of Acquired Immune & 1 \\
\hline Deficiency Syndromes & 1 \\
\hline Medicine & 1 \\
\hline International Journal of STD \& AIDS & 1 \\
\hline Medical Microbiology and Immunology & 1 \\
\hline Archives of Medical Research & 1 \\
\hline African Journal of Reproductive Health & 1 \\
\hline AlDS Patient Care and STDs & 1 \\
\hline Pesquisa Odontológica Brasileira & 1 \\
\hline Salud Pública de México & 1 \\
\hline Journal of Dental Research & 1 \\
\hline Annals Academy of Medicine Singapore & 1 \\
\hline European Journal of Medical Research & \\
\hline BMC Oral Health & 1 \\
\hline
\end{tabular}

\begin{tabular}{lc}
\hline \multicolumn{1}{c}{ Revista } & $\begin{array}{c}\text { Número } \\
\text { de } \\
\text { artículos }\end{array}$ \\
\hline HIV/AIDS & 1 \\
Journal of Applied Oral Science & 1 \\
\hline Colombia Médica & 1 \\
Cadernos de Saúde Pública & 1 \\
Journal of Medicine and Medical & 1 \\
Sciences & 1 \\
Biomédica & 52 \\
\hline Total & \\
\hline
\end{tabular}

TABLA 3

NÚMERO DE ARTÍ́CULOS POR TIPO DE ESTUDIO

\begin{tabular}{lc}
\hline \multicolumn{1}{c}{ Tipo de estudio } & Número de artículos \\
\hline Descriptivo & 25 \\
Corte transversal & 20 \\
Cohorte & 5 \\
Cohorte multicéntrico & 1 \\
Casos y controles & 1 \\
Total & $\mathbf{5 2}$ \\
\hline
\end{tabular}

TABLA 4

NÚMERO DE ARTÍCULOS POR REGIONES DEL MUNDO

\begin{tabular}{llc}
\hline & Continente & $\begin{array}{c}\text { Número de } \\
\text { artículos }\end{array}$ \\
\hline América & Norteamérica & 5 \\
& América Latina y el Caribe & 14 \\
África & 12 \\
Asia & 12 \\
Europa & 9 \\
Total & $\mathbf{5 2}$ \\
\hline
\end{tabular}

TABLA 5

NúMERO de ARTíCULOS POR REGIÓN, TIPO DE ESTUdIO, AÑo DE PUBLICACIÓN E IDIOMA

\begin{tabular}{lcccccccccc}
\hline \multirow{2}{*}{ Región } & \multicolumn{4}{c}{ Tipo de estudio } & \multicolumn{4}{c}{$\begin{array}{c}\text { Año de } \\
\text { publicación }\end{array}$} & \multicolumn{2}{c}{ Idioma } \\
\cline { 2 - 11 } & D & SC & C & CM & CyC & 2000-2005 & 2006-2010 & Inglés & Español Portugués \\
\hline $\begin{array}{l}\text { América Latina y } \\
\text { el Caribe }\end{array}$ & 6 & 7 & - & - & 1 & 4 & 10 & 8 & 4 & 2 \\
Norteamérica & 1 & - & 3 & 1 & - & 4 & 1 & 5 & - & - \\
África & 8 & 4 & - & - & - & 6 & 6 & 12 & - & - \\
Asia & 5 & 6 & 1 & - & - & 8 & 4 & 12 & - & - \\
Europa & 4 & 4 & 1 & - & - & 7 & 2 & 8 & 1 & - \\
\hline
\end{tabular}

D: descriptivo; SC: sección cruzada, C: cohorte; CM: cohorte multicéntrico; CyC: casos y controles. 
Las revistas donde se publicaron más artículos relacionados con el tema son Oral Diseases; Journal of Oral Pathology and Medicine; y Oral Surgery, Oral Medicine, Oral Pathology, Oral Radiology, and Endodontology. También se observó que múltiples revistas han publicado al menos un artículo sobre las manifestaciones orales asociadas al VIH/sida (tabla 2).

En cuanto al año de publicación, el mayor número de artículos se publicó en los años 2000, 2006 y 2008. Por el contrario en el 2005, 2006 y 2009 hubo menor producción bibliográfica sobre el tema. Los tipos de estudio de predominaron fueron descriptivos y de corte transversal. Llama la atención que en la búsqueda se encontró un estudio de casos y controles (tablas 3 y 5 ).

Con respecto a la región, se presentó la mayor producción de artículos relacionados con el tema en el continente americano, particularmente en América Latina y el Caribe, cuya producción fue mayor que la de Norteamérica. África y Asia presentaron igual cantidad de artículos y Europa fue la región con menor número de artículos (tablas 4 y 5).

En cuanto al idioma, la mayoría de los artículos se publicaron en inglés. Cabe resaltar el aumento de la producción escrita en español y portugués (tabla 5). En América Latina y el Caribe se realizaron principalmente estudios descriptivos y de corte transversal. Cabe resaltar que el único artículo de casos y controles fue efectuado en esta subregión, hubo mayor producción en el periodo 2006-2010 que en el periodo 2000-2005 y la mayoría de los artículos estaba publicada en inglés, aunque también había en español y en portugués. En Norteamérica se realizaron estudios descriptivos, de cohorte y el único de cohorte multicéntrico encontrado; hubo mayor producción en el periodo 2000-2005 que en el periodo 2006-2010 y solo se encontraron artículos en idioma inglés. Por su parte, en África se publicaron en su mayoría estudios descriptivos y algunos de corte transversal, hubo igual producción en los periodos 2000-2005 y 2006-2010 y solo se encontraron artículos en inglés. En Asia se rea- lizaron artículos descriptivos, de corte transversal y uno de cohorte, hubo mayor producción en el periodo 2000-2005 y solo se encontraron artículos en idioma inglés. Finalmente, en Europa se realizaron estudios descriptivos, de corte transversal y uno de cohorte, hubo mayor producción en el periodo 2000-2005 y solo se encontraron artículos en inglés (tabla 5).

\section{Consideraciones epidemiológicas}

A pesar de los numerosos estudios que se han realizado desde la década de los ochenta, no hubo unanimidad sobre la frecuencia de presentación de las diversas manifestaciones orales. Esto se debe a que existe amplia variación asociada al tipo de estudio, el diseño metodológico, los criterios de selección de la población estudiada (hombres, mujeres, niños, usuarios de drogas endovenosas, hombres que tienen sexo con hombres, mujeres gestantes, etc.) y el tamaño de la muestra. También dependen de la categoría de transmisión, el estadio de la infección por el $\mathrm{VIH}$, los métodos de recolección y otros factores, como el hecho de estar o no en tratamiento antirretroviral (TARV), particularmente al comparar los estudios anteriores a 1996, cuando no se disponía de dicho tratamiento, con los publicados después del 2000, cuando se considera que ha habido una expansión casi universal de los TARV.

Este hecho enfrenta al trabajador de la salud y al profesional en formación que se acerca a la literatura sobre el tema a cifras ambiguas de difícil interpretación y de poca aplicación para la población. Esto, especialmente si se considera la variabilidad en patrones de transmisión y el comportamiento clínico de la infección, que depende del área geográfica y del año de realización del estudio (39-91). Por lo anterior, y para tener un panorama global del comportamiento de las lesiones a lo largo de los años, se presenta una completa revisión de los principales estudios epidemiológicos publicados en entre el 2000 y el 2010 , realizados con población adulta, agrupados por subregiones y con sus principales implicaciones (tablas $3,4,6,7,8,9$ y 10$)$. 
TABLA 6

PreValenCia EN PORCENTAJe de LESIONES ORALES ASOCIADAS A LA INFECCIÓN POR EL VIH EN ÁFRICA

\begin{tabular}{lccccccc}
\hline \multicolumn{1}{c}{ Autores, año, país } & N & Prev. & C. O. & L. V. & E. P. & S. K. & U \\
\hline Matee et al., 2000, Tanzania (38) & 192 & 47 & 19 & 10 & - & - & 6 \\
Muzyka et al., 2001, Malawi-mujeres (39) & 138 & 14 & 1 & 6 & - & - & - \\
\hline Chidzonga, 2003, Zimbabue (40) & 156 & - & 55 & 1,3 & 8 & 19 & 11 \\
Anteyi et al., 2003, Nigeria (41) & 500 & 53 & 49 & 2,5 & 5,5 & 2,5 & 2,5 \\
\hline Adurogbanga et al., 2004, Nigeria (42) & 100 & 57 & 54 & - & 4 & - & 1,2 \\
Agbelusi et al., 2005, Nigeria (43) & 35 & - & 43 & 3 & 23 & 0 & 8 \\
\hline Obuekwe et al., 2006, Nigeria (44) & 305 & - & 78 & 6 & 10 & 3 & 11,5 \\
\hline Mbuagbaw et al., 2006, Camerún (45) & 384 & 68,8 & 35 & - & - & 9,9 & 2,1 \\
\hline Hamza et al., 2006, Tanzania (46) & 532 & 39,5 & 23,5 & 2,3 & 2,4 & 3,2 & 0,8 \\
Adedigba et al., 2008, Nigeria (47) & 225 & 84 & 73 & 1,3 & 20,5 & - & 9 \\
Bhayat et al., 2010, Sudáfrica (48) & 210 & - & 68 & 20 & 0,5 & 0,5 & - \\
Taiwo et al., 2010, Nigeria (49) & 142 & 44 & 23 & 10 & 1 & 3,5 & 3,5 \\
\hline Taiwo et al., 2010, Nigeria (50) & 278 & - & 26,2 & 10,9 & 0,7 & 2,6 & 1,5 \\
\hline
\end{tabular}

Prev.: prevalencia; C. O.: candidosis oral; L. V.: leucoplasia vellosa; E. P.: enfermedad periodontal; S. K.: sarcoma de Kaposi; U: úlceras.

TABLA 7

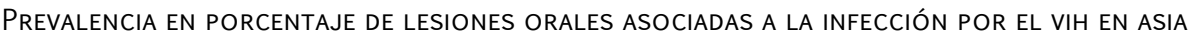

\begin{tabular}{|c|c|c|c|c|c|c|c|}
\hline Autores, año, país & $\mathbf{N}$ & Prev. & C. 0. & L. V. & E. P. & S. $K$. & $U$ \\
\hline Ranganathan et al., 2000, India (51) & 300 & 72 & 53 & 3 & 47 & - & 3 \\
\hline Khongkunthian et al., 2001, Tailandia (52) & 87 & 38 & 20,6 & 11,5 & 8 & - & 1 \\
\hline Bendick et al., 2002, Camboya (53) & 101 & 90 & 53 & 36 & 28 & - & 8 \\
\hline Nittayananta et al., 2002, Tailandia (54) & 278 & 66 & 40 & 26 & 12 & - & - \\
\hline $\begin{array}{l}\text { Reichart et al., 2003, Tailandia y } \\
\text { Camboya (55) }\end{array}$ & $\begin{array}{l}\text { Tailandia: 87; } \\
\text { Camboya: } 101\end{array}$ & $\begin{array}{l}48 \\
-\end{array}$ & $\begin{array}{l}17,2 \\
75,3\end{array}$ & $\begin{array}{l}11,5 \\
35,6\end{array}$ & $8 ; 39,6$ & & $\begin{array}{l}1 \\
8,9\end{array}$ \\
\hline Kerdpon et al., 2004, Tailandia (56) & $\begin{array}{l}\text { Norte: } 102 \\
\text { Sur: } 135\end{array}$ & $\begin{array}{l}70 \\
77\end{array}$ & $\begin{array}{l}25 \\
55\end{array}$ & $\begin{array}{l}38 \\
21\end{array}$ & $15 ; 14$ & & $\begin{array}{l}7 \\
6\end{array}$ \\
\hline Ranganathan et al., 2004, India (57) & 1000 & 86,6 & 23,8 & 2,1 & $\begin{array}{l}\text { Gingivitis: } 72,3 \text {; } \\
\text { Periodontitis: } 33,2\end{array}$ & - & 3,3 \\
\hline Jensen et al., 2005, Vietnam (58) & 170 & - & 12,4 & 5,3 & - & - & - \\
\hline Sharma et al., 2006, India (59) & 101 & - & 67 & 16 & 17 & - & 5 \\
\hline Umadevi et al., 2007, India (60) & 100 & 50 & 16 & 1 & - & - & 1 \\
\hline Nittayananta et al., 2010, Tailandia (61) & 268 & 55 & 36 & 22 & 10 & - & - \\
\hline Nittayananta et al., 2010, Tailandia (62) & $\begin{array}{l}\text { Hetero: } 186 \\
\text { IV: } 82\end{array}$ & $\begin{array}{l}75 \\
46\end{array}$ & $\begin{array}{l}44 \\
28\end{array}$ & $\begin{array}{l}33 \\
10\end{array}$ & $\begin{array}{l}12 \\
7\end{array}$ & $\begin{array}{l}- \\
-\end{array}$ & $\begin{array}{l}- \\
-\end{array}$ \\
\hline
\end{tabular}

Prev.: prevalencia; C. O.: candidosis oral; L. V.: leucoplasia vellosa; E. P.: enfermedad periodontal; S. K.: sarcoma de Kaposi; U: úlceras. 
TABLA 8

PREVALENCIA EN PORCENTAJE DE LESIONES ORALES ASOCIADAS A LA INFECCIÓN POR EL VIH EN EUROPA

\begin{tabular}{|c|c|c|c|c|c|c|c|}
\hline Autores, año, país & $\mathbf{N}$ & Prev. & C. 0 . & L. V. & E. P. & S. K. & $\mathbf{U}$ \\
\hline $\begin{array}{l}\text { Schmidt-Westhausen, et al. 2000, Alemania: } \\
\text { TARV (63) }\end{array}$ & 103 & - & 9,7 & 5,8 & 4,8 & 1 & 1 \\
\hline $\begin{array}{l}\text { Ceballos-Salobreña et al., 2000, España: TARV } \\
\text { (64) }\end{array}$ & 154 & 53,2 & 34,4 & 26,6 & 0,6 & 0 & - \\
\hline Campisi et al., 2001, Italia (65) & 136 & 47 & 19,8 & 7,4 & 4,4 & - & 3,7 \\
\hline Eyeson et al., 2002, Inglaterra (66) & 203 & 51 & 14 & 10 & 17 & 3 & 27 \\
\hline \multirow[t]{6}{*}{ Greenwood et al., 2002, Inglaterra (67) } & $\begin{array}{c}1590 \\
\text { Con TARV }\end{array}$ & & & & & & \\
\hline & 1992 & 47 & 16,2 & 44,1 & - & 2,3 & - \\
\hline & 1998 & 32,3 & 9 & 12,6 & - & 1 & - \\
\hline & Sin TARV & & & & & & \\
\hline & 1992 & 38 & 8,5 & 28,2 & - & 0 & - \\
\hline & 1998 & 37,3 & 12,8 & 17,8 & - & 1,8 & - \\
\hline \multirow[t]{4}{*}{ Nicolatou-Galitis et al., 2004, Grecia (68) } & Total 95 & & & & & & \\
\hline & Sin ART 37 & 46 & 35,1 & 8,1 & 8,1 & - & - \\
\hline & Doble ART 14 & 50 & 28,6 & 14,3 & 0 & - & - \\
\hline & PI-TARV 44 & 36,4 & 19 & 9,1 & 0 & - & - \\
\hline Kroidl et al., 2005, Alemania (69) & 139 & 86 & 7,2 & 3,6 & 76 & 0,7 & 1,4 \\
\hline Salas et al., 2006, España: TARV (70) & 90 & - & 31,1 & 1,1 & - & 3,3 & - \\
\hline Giuliani et al., 2008, Italia (71) & 200 & 26 & 24 & 12 & - & 8 & 24 \\
\hline \multirow[t]{3}{*}{ Gaitán et al., 2008, España (72) } & Total: 86 & & & & & & \\
\hline & PRE-TARV & 66,3 & 51,1 & 20,9 & - & 4 & - \\
\hline & TARV & 46,5 & 33,7 & 23,3 & - & 0 & - \\
\hline
\end{tabular}

Prev.: prevalencia; C. O.: candidosis oral; L. V.: leucoplasia vellosa; E. P.: enfermedad periodontal; S. K.: sarcoma de Kaposi; U: úlceras.

TABLA 9

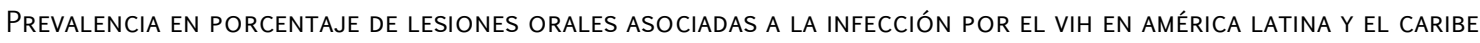

\begin{tabular}{lcccccccc}
\hline \multicolumn{1}{c}{ Autores, año, país } & N & Prev. & C. O. & L. V. & E. P. & S. K. & U \\
\hline Souza et al., 2000, Brasil (73) & 100 & - & 80,2 & 5,3 & 76,4 & 6,7 & - \\
\hline Estrada, 2001, Colombia (74) & 314 & 71 & 66 & 21 & 17 & 9 & 8 \\
\hline Ramírez-Amador et al., 2002, México (75) & 512 & 65 & 38,2 & 30,9 & - & - & - \\
\hline Ramírez-Amador et al., 2003, México (76) & 1000 & 47 & 32 & 23 & 1,7 & 2,3 & - \\
\hline Bravo et al., 2006, Venezuela (77) & 75 & 85 & 61 & 53 & 8 & 5 & 6 \\
\hline Ramírez et al., 2006, México (78) & 850 & - & 45,5 & 35,3 & 5,3 & 6,3 & 10,5 \\
\hline Casariego et al., 2006, Argentina (79) & Periodo 1: 2873 & - & 28,7 & 13,6 & 4,9 & 2,7 & 9,9 \\
& Periodo 2: 520 & - & 9,4 & 3,3 & 5,8 & 2,1 & 44,2 \\
\hline Ferreira et al., 2007, Brasil (80) & 1230 & 45 & 34 & 7,5 & 6 & 4 & 7 \\
\hline Ramírez et al., 2007, México: TARV (81) & 1134 & 42,5 & 27,7 & 17 & - & - & 6,4 \\
\hline Pedreira et al., 2008, Brasil (82) & 79 & 47 & 28 & 9 & 28 & - & - \\
\hline Pinzón et al., 2008, Colombia (83) & 319 & 77,1 & 35,7 & 13,6 & 34,2 & 3,8 & 7,5 \\
\hline Lourenço et al., 2008, Brasil (84) & 340 & 33,2 & 31,6 & 11,8 & - & 0,6 & 2,1 \\
\hline Carpio et al., 2009, Cuba (85) & 154 & 41 & 11 & 12,5 & 4,5 & - & 6 \\
\hline Gasparin et al., 2009, Brasil (86) & 300 & 39 & 59,1 & 25,2 & - & 0 & 5 \\
\hline
\end{tabular}

Prev.: prevalencia; C. O.: candidosis oral; L. V.: leucoplasia vellosa; E. P.: enfermedad periodontal; S. K.: sarcoma de Kaposi; U: úlceras. 
TABLA 10

PreValencia en Porcentaje de LeSiones orales asociadas a la INFECCIÓn POR EL VIH EN NORTEAméRICA

\begin{tabular}{|c|c|c|c|c|c|c|c|}
\hline Autores, año, país & $\mathbf{N}$ & Prev. & C. 0. & L. V. & E. P. & S. $K$. & $\mathrm{U}$ \\
\hline \multirow{3}{*}{$\begin{array}{l}\text { Patton et al., 2000, EE. UU.: terapia con inhi- } \\
\text { bidor de proteasa (87) }\end{array}$} & Total 570 & & & & & & \\
\hline & Temprana 271 & 47,6 & 20,3 & 25,8 & 4,8 & 1,1 & - \\
\hline & Tardía 299 & 37,5 & 16,7 & 11,4 & 1,7 & 0,3 & - \\
\hline Patton et al., 2000, EE. UU. (88) & 606 & 42 & 18 & 17 & 6 & 1 & 5 \\
\hline Greenspan et al., 2000, EE. UU.: mujeres (89) & 577 & - & 14 & 6 & - & 0,5 & 3 \\
\hline \multirow{3}{*}{$\begin{array}{l}\text { Greenspan et al., 2004, EE. UU.: mujeres TARV } \\
\text { (90) }\end{array}$} & 503 & & & & & & \\
\hline & PRE-TARV & - & 7,4 & 2,5 & - & - & - \\
\hline & TARV & - & 3,4 & 2,3 & - & - & - \\
\hline Owotade et al., 2008, EE. UU. (91) & 115 & - & 19 & - & - & - & 9 \\
\hline
\end{tabular}

Prev.: prevalencia; C. O.: candidosis oral; L. V.: leucoplasia vellosa; E. P.: enfermedad periodontal; S. K.: sarcoma de Kaposi; U: úlceras.

Vale la pena destacar que cada vez hay más estudios realizados en población africana que pueden encontrarse en las bases de datos de la universidad. No obstante, son insuficientes para caracterizar la diversidad de la epidemia de los países de ese continente, que es la más afectada por la pandemia, dadas las variaciones regionales en los indicadores globales de VIH y las también variadas estrategias para su contención, que pueden afectar las tasas de prevalencia de las manifestaciones orales. Los 13 estudios encontrados representan a 7 países, la mayoría de ellos en el cono surafricano, que presentan la mayor prevalencia en el ámbito global. De su estudio se pueden efectuar, con las debidas limitaciones, algunas extrapolaciones útiles para países de América Latina y el Caribe que, dos décadas después, presentan patrones epidemiológicos semejantes a los de los países de África las dos décadas pasadas (tabla 6) (38-50).

En esos estudios se resalta una prevalencia de lesiones orales cercana al $50 \%$ o superior, como es el caso de Nigeria, en el 2008, que tenía una prevalencia del 80 \%. Esta cifra suele ser presentada como la máxima alcanzada en poblaciones donde la epidemia está consolidada y para quienes aún no hay acceso universal a los servicios de salud. Una excepción notoria entre estos estudios es un estudio sobre Malawi, del cual se informa una prevalencia general del 14 $\%$ y que tiene la particularidad de ser un estudio de cohortes en mujeres con seguimiento internacional. Ello que puede sugerir un mejor acceso a los ARV y tratamientos integrales, hipótesis sugerida por la baja frecuencia de cualquiera de las lesiones estudiadas, y que difiere de otros estudios realizados en centros de atención clínica adonde acude toda la población. La no disponibilidad de datos de prevalencia desagregados por sexo no permite comparar las manifestacio- nes orales utilizando esta variable y está pendiente de efectuarse en estudios posteriores (tabla 6) (38-50).

La distribución de la prevalencia por tipo de lesión sigue los parámetros descritos en la literatura. En primer lugar, se encuentra la candidosis oral, en casos extremos como el de Nigeria, en el 2006, con una prevalencia del $78 \%$ en poblaciones que no reciben ARV; en contraste con el $19 \%$ de Tanzania o el $1 \%$ de Malawi. La leucoplasia vellosa se comporta según lo reportado; ocupa el segundo lugar con cifras que oscilan entre el 2 y el $20 \%$, lo cual sugiere que en aquellos países donde los valores son mayores hay un pobre acceso a ARV o a cuidados integrales de salud. Es particularmente alto el valor de la prevalencia del sarcoma de Kaposi en Zimbabue, en el 2003, lo cual sugiere una amplia distribución del virus del herpes humano $8(\mathrm{HH} 8)$ entre personas que viven con $\mathrm{VIH} y$, de nuevo, un pobre acceso a atención integral y TARV. Las demás lesiones (enfermedad periodontal y lesiones ulceradas) se comportan de la manera esperada y ocupan los últimos lugares en la prevalencia. Aun así, dadas las implicaciones para la calidad de vida del paciente, como en el caso de las úlceras, estas lesiones se deben tener en cuenta. Con la consolidación de la epidemia en la mayoría de países africanos y su expansión en los del África mediterránea, es de esperar en el futuro una ampliación de los países representados en los estudios disponibles (tabla 6) (38-50).

La tabla 7 reúne los estudios realizados en Asia. Se aprecia una presencia mayor de lesiones, que llega al 90 \% en Camboya. También se encontró una prevalencia del $38 \%$ en uno de los estudios de Tailandia, a pesar de que este uno de los países donde las campañas frontales para prevenir y atender a las personas con VIH son mejor implementadas. Los estudios 
disponibles representan solo a 4 países y, por esto, el análisis debe ser cuidadoso. Tener estudios comparativos, como en el caso de Tailandia, India y Camboya, es de gran valor. En general, las lesiones se comportan de acuerdo con el patrón descrito en la literatura (en particular la candidosis oral seguida de la leucoplasia vellosa), aunque se exceptúa el sarcoma de Kaposi, que no es identificado en ninguno de los estudios. Dicha información ha se ha utilizado en talleres de trabajo internacionales y puede hacer pensar en una distribución geográfica restringida del agente causal del sarcoma, el HH8, porque no se pueden hacer extrapolaciones a otras regiones donde la prevalencia de infección por el HH8 empieza a ser reportada con mayor frecuencia. Los estudios epidemiológicos de los gigantes asiáticos, India y China, modifican ampliamente las cifras consolidadas mundiales y, por lo tanto, sus datos deben manejarse con cuidado. Vale la pena estar atentos a la publicación de estudios de carácter nacional o por subregiones provenientes de estos países, pues empiezan a aparecer diagnósticos iniciales en los que se espera sean incluidas las manifestaciones orales para comprender mejor la epidemia en esta región (51-62).

Del grupo de estudios realizados en Europa (tabla 8) puede decirse que siguen el patrón esperado de prevalencia de lesiones en países del primer mundo (menores a las observadas en el tercer mundo). Solamente uno de los primeros estudios realizados en Holanda, en 1991, se describe una prevalencia general de $80 \%$, cifra que es necesario poner en contexto por el grupo de personas en que se realizó el estudio, usuarios de drogas endovenosas. Para esa época -y aun hoy- ese grupo presenta un perfil epidemiológico que refleja todas las vulnerabilidades de esta población y que se comparan con las de poblaciones de los países más pobres, donde las condiciones de vida y el acceso a salud son precarias (63-72).

Es llamativo que la información proviene de los países de Europa Occidental y queda un vacío total de los países de Europa Oriental, los cuales han experimentado un incremento en las tasas globales de VIH. Tal es el caso de Ucrania, Rusia y Moldavia. En el futuro, dichos países cambiarán seguramente el mapa epidemiológico de las manifestaciones orales observado hasta ahora. El contraste obligado de las cifras del estudio de Holanda se lleva a cabo con el estudio de Alemania, del año 2000, realizado en personas con TARV altamente efectivo y por lo cual las prevalencias de cualquiera de las lesiones alcanza sus valores más bajos. Ello corrobora que una población con ciudadanía plena y acceso universal a los sistemas de salud y atención integrales puede mantenerse prácticamente libre de complicaciones en cavidad oral asociadas con el VIH y que se reservará entonces la atención especializada para esas pocas personas que lo requieran. Ya fue señalada la deuda de información epidemiológica de los países de Europa Oriental, así como análisis discriminados por sexo, vía de transmisión, comportamiento según país de origen o tipo de terapia ARV recibida, lo que enriquecería notoriamente la comprensión global de la epidemia.

De los 19 estudios realizados en el continente americano, y a pesar de que solo están representados 6 países de América Latina y que la mayoría fueron efectuados en Estados Unidos (tablas 9 y 10), se puede deducir que las manifestaciones orales están asociadas con el nivel de desarrollo del país y el año de realización del estudio. Así, los estudios hechos en Estados Unidos muestran cifras mejores que las de los países de América Latina. Se exceptúan los realizados con población de usuarios de drogas endovenosas, en los cuales todos los casos siguen un patrón similar. Llama de nuevo la atención que los estudios realizados exclusivamente en cohortes de mujeres informan de prevalencias en promedio más bajas que aquellos conducidos con toda la población. Ese dato será de enorme interés en el futuro para la investigación sensible al género que permita dilucidar el porqué de esta baja prevalencia en estas cohortes femeninas (73-91).

México permite hacer un análisis comparativo muy interesante, ya que los estudios disponibles en las bases de datos internacionales han sido llevados a cabo por el mismo equipo de investigación, en los mismos hospitales. Ellos parecen corroborar cómo la introducción de los ARV para más población se traduce en una disminución de la prevalencia de todas las manifestaciones orales. Este hecho sirve para justificar el pleno acceso a ARV para todas las personas que viven con VIH (75-76,78,81).

De los datos descritos para el sarcoma de Kaposi llama notoriamente la atención que Colombia, en el 2001, era el país que mostraba mayores tasas en la región con un $9 \%$. Esta cifra se explica porque los datos corresponden a pacientes que no recibían TARV de ningún tipo y pertenecían en esa época al mal llamado grupo de los vinculados al sistema de salud y que recibían atención médica para el VIH, pero no ARV. Tal situación hoy, por fortuna, ha cambiado. Las cifras 
de los estudios de Colombia, Brasil, Venezuela y Cuba deben tomarse con precaución, dado el tamaño de sus muestras de estudio, pero representan un avance notorio en la investigación epidemiológica, toda vez que algunos años atrás, a pesar de saber que se realizaba investigación sobre manifestaciones orales en muchos países, esta no se publicaba en revistas internacionales o indizadas y, por lo tanto, no se encontraba en las bases de datos información de estos países. Se empieza poco a poco a ganar en visibilidad, pero aún existe una deuda para los países de América Central y el Caribe y el resto de Suramérica. Asimismo, existe el reto de realizar estudios de alcance nacional donde se incluyan las manifestaciones orales como variable de análisis $(74,83)$.

En Colombia existen varios estudios de morbilidad asociada a la infección por el $\mathrm{VIH}$, que no aparecen en las bases de datos internacionales. Sin embargo, por su relevancia para la comunidad científica, se mencionan aquí. Por un lado, está el estudio de Prada y colaboradores (92), quienes indicaron una prevalencia del 17,6 \% para la candidosis oral y esofágica, y del 5,1\% para la leucoplasia vellosa entre 244 pacientes $\mathrm{VIH}+$ examinados en Bogotá. Otro estudio es el de Velásquez y colaboradores (93), en Medellín, donde estudiaron 193 pacientes en estadio sida (clasificación CDC de 1987) e informaron de una prevalencia de candidosis del 78 \%. También en el departamento del Atlántico, Villanueva y colaboradores (94), en 138 pacientes con infección por $\mathrm{VIH}$, informaron de una prevalencia de candidosis oral y esofágica del 18,1 \%. Finalmente, en el Hospital San Juan de Dios de Bogotá, Estrada $(5,95)$ informó prevalencias elevadas para todas las lesiones teniendo en cuenta que la población allí atendida correspondía a la más vulnerable y con escasas posibilidades de recibir medicamentos ARV.

\section{CONCLUSIONES}

Se ha presentado a los lectores el panorama más completo posible de los estudios disponibles sobre manifestaciones orales del $\mathrm{VIH}$, provenientes de todo el mundo, agrupados por subregiones. Ellos constituyen una aproximación a la prevalencia global de manifestaciones orales y la distribución de las 5 principales lesiones, donde estaba la información y si los pacientes recibían o no TARV. Queda un vacío en cuanto a cantidad y calidad de la información de muchos países de los que se conoce la tasa global de HIV; pero nada referente a las manifestaciones orales de la población afectada por la epidemia. Se necesita información de Europa Oriental, África del Norte, Oceanía, América Central y el Caribe, así como de Asia Central, para poder completar el panorama epidemiológico mundial y realizar mejores contrastaciones.

A pesar de limitada información, algunas tendencias generales se pueden identificar al final del estudio. Ellas incluyen el comportamiento similar con respecto al orden de frecuencia de las manifestaciones en todo el mundo (con las diferencias ya mencionadas en cada subregión); la marcada diferencia del patrón de lesiones en los países desarrollados frente a aquellos en vías de desarrollo en los años revisados; diferencias notables entre países y en su interior cuando se revisa la vía de transmisión, pues hay un peor comportamiento de las lesiones relacionadas con el uso de drogas endovenosas que con la vía de transmisión sexual, y mejor comportamiento de las lesiones en estudios realizados exclusivamente con mujeres. Este último hallazgo abre el camino a futuras investigaciones que permitan determinar si realmente existen diferencias entre hombres y mujeres, pues no hay estudios informados solo en hombres, o si se trata de un sesgo de selección de los estudios. Por último, se encontró una disminución sustancial en las prevalencias después de la introducción y extensión de los ARV en todos los estudios revisados.

Estos resultados, relevantes para el diagnóstico de la situación de morbilidad oral asociada al VIH en el mundo, son aún insuficientes en la caracterización epidemiológica precisa de la población. Por lo tanto, es necesario realizar estudios descriptivos, longitudinales y transversales (y ojala multicéntricos) que superen los vacíos metodológicos detectados y comentados en esta revisión. Tales estudios deberán incluir análisis de género. Tener la información del comportamiento de las lesiones en el mundo permitirá contrastar la situación de cada país en cuanto a tipo de desarrollo y de epidemia, con el fin de establecer políticas públicas en salud oral y de educación, que sean aplicables a la formación del talento humano en salud y a la organización y prestación de servicios odontológicos integrales y de calidad para las personas que conviven con el VIH. Se espera que el presente análisis de la literatura epidemiológica oral relevante en español motive a estudiantes y profesionales de distintas áreas a profundizar en el estudio de las manifestaciones orales asociadas al VIH y, en lo posible, a emprender estudios en las poblaciones que son sujeto de acciones en salud. 


\section{REFERENCIAS}

1. Fast PE, McNamara JG. Research and development for a vaccine to prevent HIV infection and AIDS. En: Polsky BW, Clumeck N, Armstrong D, Cohen J. HIV and AIDS: infectious diseases. London: Mosby-Harcourt publishers; 1999. pp. 4.1-4.4.

2. Kaplan JE, Hanson D, Dworkin MS, Frederick T, Bertolli J, Lindegren ML, Holmberg S, Jones JL. Epidemiology of human immunodeficiency virus associated opportunistic infections in the United States in the era of highly active antiretroviral therapy. Clin Infect Dis. 2000; 30 (Suppl 1): S5-S14.

3. Onusida. Informe sobre la epidemia mundial de VIH/ SIDA. Ginebra: ONUSIDA; 2009.

4. Onusida, República de Colombia, Ministerio de Salud. Infección por VIH y SIDA en Colombia: aspectos fundamentales, respuesta nacional y situación actual. Un balance histórico hacia el nuevo siglo. Bogotá: Onusida, Ministerio de Salud; 2001.

5. Estrada JH. Lesiones orales asociadas con la infección por VIH/Sida en individuos sin tratamiento antirretroviral en el Hospital San Juan de Dios de Bogotá. Biomédica. 2001; 21: 333-44.

6. Greenspan JS. Sentinels and signposts: the epidemiology and significance of the oral manifestations of HIV disease. Oral Dis. 1997; 3(Suppl I): S13-S17.

7. Greenspan JS, Barr CE, Sciubba JJ, Winkler JR. Oral manifestations of HIV infection. Definitions, diagnostic criteria and principles of therapy. Oral Surg Oral Med Oral Pathol 1992; 73: 142-4.

8. Greenspan D, Greenspan JS. Oral manifestations of human immunodeficiency virus infection. Dental Clinics North Am. 1993; 37: 21-32.

9. Glick M. The role of the dentist in the era of AIDS. Dent Clin North Am. 1996; 40: 343-57.

10. Greenspan D, Greenspan JS. Management of the oral lesions of HIV infection. J Am Dent Assoc. 1991; 122: 26-32.

11. Scully C, McCarthy G. Management of oral health in persons with HIV infection. Oral Surg Oral Med Oral Pathol. 1992; 73: 215-25.

12. American Dental Association. Dental treatment to the HIV infected patients. J Am Den Assoc; 1995; 126(Suppl 1): 39.

13. República de Colombia, Ministerio de Salud, Dirección General de Promoción y Prevención. Manual de aspectos básicos: infección por VIH/SIDA-hepatitis B. Medidas para el control de infecciones en Odontología. Bogotá: Ministerio de Salud; 1995. p. 42.

14. USPH/IDSA Prevention of Opportunistic Infections Working Group. 1999 USPH/IDSA guidelines for prevention of opportunistic infections in persons infected with human immunodeficiency virus. Clin Infect Dis. 2000; 30(Suppl 1): S29-S65.

15. Organización Panamericana de la Salud (OPS). Sistemas y servicios de salud: programa regional de salud buco- dental. Estrategia regional de salud bucodental para los años noventa. Quito: OPS; 1994.

16. Reidpath D, Chan K. HIV, Stigma, and rates of infection: a rumour without evidence. PLoS Med. 2006; 3(10): 1708-10.

17. Parker R, Aggleton P. HIV and AIDS-related stigma and discrimination: a conceptual framework and implications for action. Soc Sci \& Med. 2003; 57: 13-24.

18. Pindborg JJ. Classification of oral lesions associated with HIV infection/AIDS. Oral Surg Oral Med Oral Pathol. 1989; 67: 292-5.

19. Scully C, Laskaris G, Pindborg J, Porter SR, Reichart P. Oral manifestations of HIV infection and their management. I. More common lesions. Oral Surg Oral Med Oral Pathol. 1991; 71: 158-66.

20. Scully C, Laskaris G, Pindborg J, Porter SR, Reichart P. Oral manifestations of HIV infection and their management. II. Less common lesions. Oral Surg Oral Med Oral Pathol. 1991; 71: 167-71.

21. Scully C, Bagg J. Viral infections in dentistry. Oral Maxillofac Surg Infect. 1992; 111: 102-15.

22. Classification and diagnostic criteria for oral lesions in HIV infection. EC-Clearinghouse on Oral Problems Related to HIV Infection and WHO Collaborating Centre on Oral Manifestations of the Immunodeficiency Virus. J Oral Pathol Med. 1993; 22: 289-91.

23. Porter S, Scully C. HIV: the surgeon's perspective. Part 2. Diagnosis and management of non-malignant oral manifestations. Br J Oral Maxillofac Surg. 1994; 32: 231-40.

24. Greenspan D, Greenspan JS. HIV related disease. Lancet. 1996; 348: 729-33.

25. Casiglia J, Woo S. Oral manifestations of HIV infection. Clin Dermatol. 2000; 18: 541-51.

26. Plettenberg A, Reisinger E, Lenzner $U$, Listemann $H$, Ernest M, Kern P, Dietrich M, Meigel W. Oral candidosis in HIV infected patients: prognostic value and correlation with immunological parameters. Mycoses. 1990; 5: 610-8.

27. Nielsen H, Bentsen KD, Hojtved L, Willemoes EH, Scvheutz F, Schiødt M, Stoltze K, Pindborg JJ. Oral candidiasis and immune status of HIV infected patients. J Oral Pathol Med. 1997; 26: 237-43.

28. Estrada JH, Núñez GA, Rodríguez M, Díaz D. Atención al paciente con VIH. Guía de Práctica Clínica Basadas en la Evidencia. Bogotá: Asociación Colombiana de Facultades de Odontología, Instituto del Seguro Social; 1998.

29. Husak R, Garbe C, Orfanos C. Oral hairy leukoplakia in 71 HIV seropositive patients: clinical symptoms, relation to immunologic status and prognostic significance. J Am Acad Dermatol. 1996; 35: 928-34.

30. Triantos D, Porter SR, Scully C, Gee C. Oral hairy leukoplakia: clinicopathologic features, pathogenesis, diagnosis and clinical significance. Clin Infect Dis. 1997; 25: 1392-6.

31. Pedra E, Dias EP, Rocha ML, Silva Júnior A, Spyrides KS, Ferreira SM, Polignano GA, Feijó EC, Da Fonseca EC. Oral hairy leukoplakia: histopathologic and cytopathologic 
features of a subclinical phase. Am J Clin Pathol. 2000; 114: 395-401.

32. Yeung SC, Stewart GJ, Cooper DA, Sindhusake D. Progression of periodontal disease in HIV seropositive patients. J Periodontol. 1993; 64: 651-7.

33. Glick M, Muzyka BC, Salkin LM, Lurie D. Necrotizing ulcerative periodontitis: a marker for immune deterioration and a predictor for the diagnosis of AIDS. J Periodontol. 1994; 65: 393-7.

34. Robinson PG. Which periodontal changes are associated with HIV infection? J Clin Periodontol. 1998; 25: 278-85.

35. Lamster IB, Grbic JT, Mitchell-Lewis D, Begg MD, Mitchell A. New concepts regarding the pathogenesis of periodontal disease in HIV infection. Ann Periodontol. 1998; 3: 62-75.

36. Estrada JH. Enfermedad periodontal asociada al VIH. CD Multimedia. Bogotá: Universidad Nacional de Colombia; 2006.

37. Moore P, Chang Y. Kaposi's sarcoma (KS); KS-associated herpesvirus and the criteria for causality in the age of molecular biology. Am J Epidemiol. 1998; 147(3): 217-21.

38. Grogg KL, Miller RF, Dogan A. HIV infection and lymphoma. J Clin Pathol. 2007; 60: 1365-72.

39. Matee Ml, Scheutz F, Moshy J. Occurrence of oral lesions in relation to clinical and immunological status among HIV-infected adult Tanzanians. Oral Dis. 2000; 6, 106-11.

40. Muzyka BC, Kamwendo L, Mbuweza E, Lopez NB, Glick M, Matheson PB, Kershbaumer R, Nyrienda T, Malamud D, Constantine NT, Thompson J, Nyasulu Y, Saville R, Berthold P. Prevalence of HIV-1 and oral lesions in pregnant women in rural Malawi. Oral Surg Oral Med Oral Pathol Oral Radiol Endod. 2001; 92: 56-61.

41. Chidzonga MM. HIV/AIDS orofacial lesions in 156 Zimbabwean patients at referral oral and maxillofacial surgical clinics. Oral Dis. 2003; 9(6): 317-22.

42. Anteyi KO, Thacher TD, Yohanna S, Idoko Jl. Oral manifestations of HIV-AIDS in Nigerian patients. Int J STD AIDS. 2003; 14(6): 395-8.

43. Adurogbanga Ml, Aderinokum GA, Odaibo GN, Olaleye OD, Lawoyin TO. Oro-facial lesions and CD4 counts associated with HIV/AIDS in adult population in Oyo state, Nigeria. Oral Dis. 2004; 10: 319-26.

44. Agbelusi GA, Wright AA. Oral lesions as indicators of HIV infection among routine dental patients in Lagos, Nigeria. Oral Dis. 2005; 11: 370-3.

45. Obuekwe ON, Onunu AN. Gender and oral manifestations of HIV infection among adult Nigerians. Rev Afr Santé Reprod. 2006; 10(2): 81-9.

46. Mbuagbaw J, Eyong I, Alemnji G, Mpoudi N, Same-Ekobo A. Patterns of skin manifestations and their relationships with CD4 counts among HIV/AIDS patients in Cameroon. International J Dermatol. 2006; 45: 280-4.

47. Hamza JM, Matee IN, Simon NM, Kikwilu E, Moshi J, Mugusi F, Mikx HM, Verweij E, Ven JAM. Oral manifestations of HIV infection in children and adults receiving highly active anti-retroviral therapy [HAART] in Dar es Salaam, Tanzania. BMC Oral Health. 2006, 6: 12.
48. Adedigba MA, Ogunbodede EO, Jeboda SO, Naidoo S. Patterns of oral manifestation of HIV/AIDS among 225 Nigerian patients. Oral Dis. 2008; 14: 341-6.

49. Bhayat A, Yengopal V, Rudolph M. Predictive Value of group I oral lesions for HIV infection. Oral Surg Oral Med Oral Pathol Oral Radiol Endod. 2010; 109: 720-3.

50. Taiwoo OO, Zuwaira H. The impact of highly active antiretroviral therapy (HAART) on the clinical features of HIV-related oral lesions in Nigeria. AIDS Res Ther. 2010; 7: 19-24.

51. Taiwoo OO, Zuwaira H. HIV-related oral lesions as markers of immunosuppression in HIV sero-positive Nigerian patients. J Med Sci. 2010; 1(5): 166-170.

52. Ranganathan K, Reddy BVR, Kumarasamy N, Solomon S, Viswanathan R, Kohnson NW. Oral lesions and conditions associated with human immunodeficiency virus infection in 300 south Indian patients. Oral Dis. 2000; 6: 152-7.

53. Khongkunthian P, Grote M, Isaratanan W, Piyaworawong S, Reichart P. Oral manifestations in HIV-positive adults from Northern Thailand. J Oral Pathol Med. 2001; 30: 220-3.

54. Bendick C, Scheifele C, Reichart PA. Oral manifestations in 101 Cambodian patients with HIV infection and AIDS. J Oral Pathol Med. 2002: 31: 1-4.

55. Nittayananta W, Chanowanna N, Winn T, Silpapojakul K, Rodklai A, Jaruratanasirikul S, Liewchanpatana K. Coexistence between oral lesions and opportunistic systemic diseases among HIV-infected subjects in Thailand. J Oral Pathol Med. 2002; 31: 163-8.

56. Reichart P.A, Khongkhunthian P, Bendick C. Oral manifestations in HIV-infected individuals from Thailand and Cambodia. Med Microbiol Immunol 2003; 192: 157-60.

57. Kerdpon D, Pongsiriwet S, Pangsomboon K, lamaroon A, Kampoo K, Sretrirutchai S, Geater A, Robison V. Oral manifestations of HIV infection in relation to clinical and CD4 immunological status in northern and southern Thai patients. Oral Dis. 2004; 10: 138-44.

58. Ranganathan K, Umadevi M, Saraswathi TR, Kumarasamy N, Solomon S, Johnson N. Oral lesions and conditions associated with human immunodeficiency virus infection in 1000 South Indian patients. Ann Acad Med Singapore. 2004; 33(Suppl): 37-42.

59. Jensen TO, Tam VV, Mai NT, Ut do Q, Dat DD, Lien NT, Nga NT, Bygbjerg IC. Oral and constitutional manifestations of HIV-infected hospital patients in Northern Vietnam. Southeast Asian J Trop Med Public Health. 2005; 36(6): 1459-68.

60. Sharma G, Pai, KM, Suhas S, Ramapuram JT, Doshi D, Anup N. Oral manifestations in HIV-AIDS infected patients from India. Oral Dis. 2006; 12: 537-42.

61. Umadevi KM, Ranganathan K, Pavithra S, Hemalatha R, Saraswathi TR, Kumarasamy N, Solomon S, Greenspan J. Oral lesions among persons with HIV disease with and without highly active antiretroviral therapy in southern India. J Oral Pathol Med. 2007; 36: 136-41. 
62. Nittayananta W, Talungchit S, Jaruratanasirikul S, Silpapojakul K, Chayakul P, Nilmanat A, Pruphetkaew N. Effects of long-term use of HAART on oral health status of HIV-infected subjects. J Oral Pathol Med. 2010; 39: 397-406.

63. Nittayananta W, Chanowanna N, Winn T. Mode of HIV transmission associated with risk of oral lesions in HIVinfected subjects in Thailand. J Oral Pathol Med. 2010; 39: 195-200.

64. Schmidt-Westhausen A, Priepke F, Bergmann F, Reichart $P$. Decline in the rate of oral opportunistic infections following introduction of highly active antiretroviral therapy. J Oral Pathol Med. 2000; 29: 336-41.

65. Ceballos-Solobreña A, Gaitán-Cepeda L, Ceballos-García L, Lezama-Del Valle D. Oral Lesions in HIV/AIDS Patients undergoing highly active antiretroviral treatment including protease inhibitors: a new face of oral AIDS? AIDS Patient Care STDS. 2000; 14(12): 627-35.

66. Campisi G, Pizzo G, Mancuso, Margiotta V. Gender differences in human immunodeficiency virus-related oral lesions: An Italian study. Oral Surg Oral Med Oral Pathol Oral Radiol Endod. 2001; 91: 546-51.

67. Eyeson JD, Tenat-Flowers M, Cooper DJ, Johnson NW, Warnakulasuriya KAAS. Oral manifestations of an HIV positive cohort in the era of highly active anti-retroviral therapy (HAART) in south London. J Oral Pathol Med. 2002: 31: 169-74.

68. Greenwood I, Zakrzewska JM, Robinson PG. Changes in the prevalence of HIV-associated mucosal disease at a dedicated clinic over 7 years. Oral Dis. 2002; 8: 90-4.

69. Nicolatou-Calitis O, Velegraki A, Paikos S, Economopoulou $P$, Stefaniotis T, Papanikolaou IS, Kordossis T. Effect of PI-HAART on the prevalence of oral lesions in HIV-1 infected patients. A Greek study. Oral Dis. 2004; 10: $145-50$.

70. Kroidl A, Schaeben A, Oette M, Wettstein M, Herfordt A, Häussinger D. Prevalence of oral lesions and periodontal diseases in HIV-infected patients on Antiretroviral therapy. Eur J Med Res. 2005; 10: 448-53.

71. Salas E, Küstner E, López J, Llabrés X, Rivera I. Efecto de los tratamientos antirretrovirales en las manifestaciones orales de los pacientes $\mathrm{VIH+}$. Av Odontoestomatol. 2006; 22(6): 315-26.

72. Giuliani M, Lajolo C, Sartorio A, Ammassari A, Lacaita MG, Scivetti M, Tamburrini E, Tumbarello M. Oral lesions in HIV and HVC co-infected individuals in HAART era. J Oral Pathol Med. 2008; 37: 468-474.

73. Gaitán LA, Ceballos A, López K, Arzate N, Jiménez Y. Oral lesions and immune reconstitution syndrome in HIV+/AIDS patients receiving highly active antiretroviral therapy. Epidemiological evidence. Med Oral Patol Oral Cir Bucal. 2008 Feb 1; 13(2): 85-93.

74. Souza LB, Pereira L, Medeiros AC, Araújo R, Mesquita OJ. Oral manifestations in HIV. Infected patients in a Brazilian population. Pesqui Odontol Bras. 2000; 14(1): 79-85.

75. Ramírez-Amador V, Esquivel-Pedraza L, Irigoyen-Camacho E, Anaya-Saavedra G, González-Ramírez I. Asocia- ción de lesiones bucales con el estado serológico para el VIH. Salud Publ Mex. 2002; 4(2): 87-91.

76. Ramírez-Amador V, Esquivel-Pedraza L, Sierra-Madero J, Anaya-Saavedra G, González-Ramírez I, Ponce-de-León $\mathrm{S}$. The changing clinical spectrum of human immunodeficiency virus (HIV)-related oral lesions in 1,000 consecutive patients. A 12 year study in a referral center in Mexico. Medicine. 2003; 82(1): 39-50.

77. Bravo IM, Correnti M, Escalona L, Perrone M, Brito A, Tovar V, Rivera H. Prevalence of oral lesions in HIV patients related to CD4 cell count and viral load in a Venezuelan population. Med Oral Patol Oral Cir Bucal. 2006; 11: E33-9.

78. Ramírez-Amador V, Anaya-Saavedra G, Calva J, Pérezde-Corcho T, López-Martínez C, González-Ramírez I, Sierra-Madero J. HIV-related oral lesions, demographic factors, clinical staging and anti-retroviral use. Arch Med Res. 2006; 37: 646-54.

79. Casariego Z, Fonio S, Micinquevich S. Lesiones estomatológicas en pacientes $\mathrm{VIH}-1$ reactivos: comparación de proporción en dos poblaciones independientes. Av Odontoestomatol. 2006; 22(4): 217-22.

80. Ferreira S, Noce C, Silva A, Goncalves L, Torres S, Meeks V, Luiz R, Dias E. Prevalence of oral manifestations of HIV infection in Rio De Janeiro, Brazil from 1988 to 2004. AIDS Patient Care STDS. 2007; 10: 724-31.

81. Ramírez-Amador V, Ponce S, Anaya-Saavedra G, Crabtree B, Sierra-Madero J. Oral lesions as clinical markers of highly active antiretroviral therapy failure: a nested case-control study in Mexico City. HIV/AIDS 2007; 45: 925-32.

82. Pedreira EN, Cardoso CL, Barroso E, Santos JA, Fonseca FP, Taveira LA. Epidemiological and oral manifestations of HIV-positive patients in a specialized service in Brazil. J Appl Oral Sci. 2008; 16(6): 369-75.

83. Pinzón EM, Bravo SM, Méndez F, Clavijo GM, León ME. Prevalencia y factores relacionados con la presencia de manifestaciones orales en pacientes con VIH/SIDA, Cali, Colombia. Colomb Med. 2008; 39: 346-55.

84. Lourenço AG, Moraes LT. Oral lesions in HIV infected individuals from Ribeirão Preto, Brazil. Med Oral Patol Oral Cir Bucal. 2008 May 1; 13(5): 281-6.

85. Carpio E, López V, Fardales V, Benítez I. Oral manifestations of HIV infection in adult patients from the province of Sancti Spiritus, Cuba. J Oral Pathol Med. 2009; 38: 126-31.

86. Gasparin AB, Vargas F, Cademartori C, Mendoza-Sassi RA, Silveira J, Barral AM, Zhang L, Cesar J. Prevalence of oral lesions in persons with HIV and associated factors in a southern Brazilian city. Cad Saúde Pública. 2009; 25(6): 1307-15.

87. Patton L, McKaig R, Strauss R, Rogers D, Eron J, Hill C. Changing prevalence of oral manifestations of human immunodeficiency virus in the era of protease inhibitor therapy. Oral Surg Oral Med Oral Pathol Oral Radiol Endod. 2000; 89: 299-304.

88. Patton LL, Hill C. Sensitivity, specificity, and positive 
predictive value of oral opportunistic infections in adults with HIV/AIDS as markers of immune suppression and viral burden. Oral Surg Oral med Oral Pathol Oral Radiol Endod. 2000; 90: 182-8.

89. Greenspan D, Komaroff E, Redford M, Phelan J, Navazesh M, Alves ME, Kamrath H, Mulligan R, Barr CE, Greenspan JS. Oral mucosal lesions and HIV viral load in the women's interagency HIV study (WHIS). J Acquir Immun Defic Syndr. 2000; 25(1): 44-50.

90. Greenspan D, Gange SJ, Phelan JA, Navazesh M, Alves MEAF, MacPhail LA, Mulligan R, Greenspan JS. Incidence of oral lesions in HIV-1-infected women: reduction with HAART. J Dent Res. 2004; 83(2): 145-50.

91. Owotade FJ, Shiboski CH, Poole L, Ramstead CA, Malvin K, Hecht FM, Greenspan JS. Prevalence of oral disease among adults with primary HIV infection. Oral Dis. 2008; 14: 497-99.

92. Prada G, Torres A, García F, Plata A, De Merino N. Infección por VIH-1. Análisis de 244 casos. Acta Med Colomb. 1992; 17: 376-82.

93. Velásquez G, Betancur J, Estrada S, Nagles J, Orozco B, Ospina S, Panneso R, Restrepo A, Robledo J. Infecciones observadas en 193 pacientes con sida. Estudio multicéntrico en la ciudad de Medellín 1987-92. Acta Med Colomb. 1993; 18: 56-65.

94. Villanueva A, Maquire J, Ramírez AR, Hernández T. Problemas en el manejo de pacientes con sida. Biomédica 1994; 14: 5-15.

95. Estrada JH. Manifestaciones orales asociadas a la infección por VIH/ sida en 123 pacientes del Hospital San Juan de Dios de Bogotá. Rev Fac Med Univ Nac Colomb. 1995; 43: 188-93.

\title{
CORRESPONDENCIA
}

\author{
John Harold Estrada Montoya \\ jhestradam@unal.edu.co \\ jhestradam@gmail.com \\ Diego Alejandro Ramírez Rojas \\ daramirezr@unal.edu.co
}


